\title{
The Emulation of Social Institutions as a Method of Coevolution
}

\author{
Deborah Vakas Duong \\ Science Applications International Corporation \\ 6359 Walker Lane, Suite 100 \\ Alexandria, Virginia 22310 \\ (703)253-1914 \\ deborah.v.duong@saic.com
}

\author{
John Grefenstette \\ George Mason University \\ 10900 University Blvd, MS 5B3 \\ Manassas, VA 20100 \\ (703)993-8398 \\ jgrefens@gmu.edu
}

\begin{abstract}
This paper offers a novel approach to coevolution based on the sociological theory of symbolic interactionism. It provides a multi-agent computational model along with experimental results that suggest improved fitness, robustness, and knowledge due to emergent symbol systems. The main contribution of the symbolicinteractionist approach to coevolution is the concept of the emergence of a system in the abstract, where an interface between agents evolves. The interface is an emergent symbol system that focuses selective pressure among agents in ways that have been beneficial to agents as a whole in the past, creating a coevolving system that takes advantage of epistasis rather than having to prevent it. Global fitness thereby emerges from local, selfish interaction. The assignment of roles in this system is endogenous.
\end{abstract}

\section{Categories and Subject Descriptors}

I.2.11 [Artificial Intelligence]: Distributed Artificial Intelligence - intelligent agents, multi-agent systems, coherence and coordination.

I.2.6 [Artificial Intelligence]: Learning - induction.

\section{General Terms}

Algorithms

\section{Keywords}

Evolutionary Computation, Cooperative Coevolution, MultiAgent Systems, Social Science Simulation

\section{INTRODUCTION}

This paper introduces Symbolic Interactionist Coevolution, a method of coevolution based on social theory. Coevolving genetic algorithms form a system as in cooperative coevolution [1], however, the roles performed by individual GAs in this system emerge endogenously through epistatic interactions mediated through communication. The key difference between the symbolic interactionist method of coevolution and standard

Copyright is held by the author/owner(s). GECCO'05, June 25-29, 2005, Washington, DC, USA. ACM 1-59593-010-8/05/0006. cooperative coevolution is that in symbolic interactionist coevolution, an abstract version of a system of GAs is created through the evolution of the communicative interface between the individual GAs. The emergent roles are the abstract classes of the system, and agents, which each hold an individual GA of the system, become instantiations of these classes through a process of symbolic interaction. Many agents may occupy the same role, as opposed to the placement of individual GAs into separate roles that occurs in cooperative coevolution.

The formation of the abstract system is achieved through a simulation of social processes from the field of sociology -especially symbolic interactionism. The symbolic interactionist method of coevolution uses as its model the formation of upper level social behavioral patterns (institutions) from lower level symbolic interaction. Symbolic interactionism emphasizes that human interactions are based on the interpretation of the meanings of signs, and that a symbol system mediates all human interaction. Both interpreters and displayers of signs simultaneously seek meaning in signs, and through this lower level process, upper level institutions emerge, and a consensus on the meaning of signs forms. Symbolic interactionist coevolution emulates the evolution of this mediating symbol system. In symbolic interactionist coevolution, the channels for influence of the species on one another itself evolves, as epistatic selective pressure is mediated through the evolved symbol system. The way in which individual GAs relate to each other itself evolves. This evolving interface between agents contains within it the experiences of past agents and the cumulative expression of their needs. This interface of communication is created through the consensus of many individual decisions, forming a system that is good for the whole. A global goal is reached in the form of institutions that emerge from local social interactions.

\section{ESSENTIAL ELEMENTS}

The requirements for the creation of a symbolic-interactionist system are listed below. They apply whether the system being created is for computational social science (learning about social phenomena through simulation), or for AI (using social processes to solve a problem). These ingredients turn a group of agents into a social system, complete with emergent social institutions to govern their interactions with each other. The use of evolutionary computation techniques as opposed to other methods of induction is not a requirement. 
1. Individual agents each have their own private, unseeded method of induction.

2. Agents communicate with each other through the display and reading of signs (for example, tags).

3. The meanings of signs are induced both by the displayer and by the reader ("double induction").

4. An agent is closed with respect to meaning: it can only interpret through its own inductions, and can not copy another agent's interpretations.

5. The meanings of signs are induced on the basis of economic advantage that an interpretation brings the agent, on an individual basis.

6. Agents have a small economy where either goods or services are traded, and the fitness of interpretations of signs is based either on the utility of goods or services that are received through barter, or the amount of money obtained if they have a money economy.

7. The signs denote either the good (or service) traded; or the seller of the good (or service).

8. The good or service may be a solution to a problem, or a partial one. For example, it may be an encoding of primitive actions to take or a way to use an AI program.

9. Goods may be composed of other goods, services may employ the services of others.

When agents are designed in this way, they form networks of trading relations and the signs that they display will come to hold knowledge of the relations between problem solving parts, in the form of an interface between the agents. The parts are emergent roles in a system that solves a problem. For example, symbolicinteractionist coevolution could be applied the game "Go." Agents could employ other agents to perform tasks, or do the tasks themselves. They could band together in hierarchies created by employment, and compete in tournaments with each other. The lowest level of the hierarchy would have agents learning a pattern on the board, to detect or move into.

Symbolic Interactionist Coevolution has been applied to problems in computational social science. In SISTER, a Symbolic Interactionist Simulation of Trade and Emergent Roles, the problem is to form the networks of trade needed to create composite goods (that is, goods made of other goods). It is a problem of social coordination. This program successfully demonstrates how symbolic interaction recreates a system of roles. Agents display and read signs to know who to trade with, and these signs come to mean sets of behaviors or roles. For example, an agent may display a sign " 0101 " that other agents come to interpret as meaning "I make and sell succotash." It comes to have this meaning in that, when an agent displays the "0101", other agents ask that agent to buy the ingredients of succotash and to sell succotash. Knowledge of how to make the composite good is distributed in the expectations that agents come to have of the displayers of a sign. For example, if a new agent in the simulation displays the sign for succotash without knowing how to cook it, it would quickly learn the ingredients from marketers who ask agents displaying sign " 0101 " to buy the ingredients for succotash. It has been shown that the knowledge of society, as encoded in the symbol system, is retained even through a complete overturn of agents through death and rebirth. New agents learn about roles as they learn the symbol system of their society, roles which transcend the lives of individual agents, who merely instantiate a role [2].

\section{EVOLVING INTERFACES}

This model of the spread of culture is useful as a general artificial intelligence technique. Using this technique, we have evolved a system in the abstract by evolving the interface between the parts. The abstractness of the system is advantageous because it allows many agents within each emergent role. First of all, it is good because we do not have to choose the number of agents to include in the system before it has formed, or use death to select them out, as in cooperative coevolution. If there are more agents than parts, then the agents can double up and take on the same role.

Evolving a system in the abstract is advantageous because, in the process of growing the interface between abstract agent classes, we accumulate the experiences of agents that have participated in similar interactions. The knowledge gained by agent experiences is held in the expectations that agents have of the signs, as in the succotash example above. By holding knowledge of relations in the expectations that individual agents have of induced symbols, we allow an accumulation of knowledge over time. The "interface" of symbols, which mediates selective pressure of agents on each other, is created through the consensus of many agents.

Through learning this interface, as in the succotash example, one agent can take advantage of the experiences of other agents, and then add to them. Agents create each other through these mutual expectations, and regulate the epistasis of coevolution so that they create each other in ways that are advantageous to all of the agents. Selective pressures of one agent on another are focused in ways that are good for the whole, because their interactions with each other have evolved with contributions of selective pressure from many agents acting in their self interest. Thus the consensus approaches a global optimum, emerging from the selfish individual actions of agents. This creation of each other by self fulfilling prophecy, through a double induction on signs is based on social theory. Through this symbolic interaction of expectations, institutions that are globally good for the agents (such as the role-based division of labor in SISTER) emerge.

\section{ACKNOWLEDGEMENTS}

The authors thank Claudio Cioffi, Kenneth DeJong, Harold Morowitz, Larry Hunter, Tom Dietz and Erik Davis for their valuable comments and their support of this project.

\section{REFERENCES}

[1] Potter, Mitchell and Kenneth DeJong. "Cooperative Coevolution: An Architecture for Evolving Coadapted Subcomponents." Evolutionary Computation, 8(1), 2000,129.

[2] Duong, Deborah and John Grefenstette. "SISTER: A Symbolic Interactionist Simulation of Trade and Emergent Roles." JASSS 8(1), 2005. http://jasss.soc.surrey.ac.uk/8/1/1.html 